TITLE:

\title{
Signed ring families and signed posets
}

$\operatorname{AUTHOR}(S)$ :

Ando, Kazutoshi; Fujishige, Satoru

CITATION:

Ando, Kazutoshi ...[et al]. Signed ring families and signed posets.

Optimization Methods and Software 2021, 36(2-3): 262-278

ISSUE DATE:

2021

URL:

http://hdl.handle.net/2433/262767

\section{RIGHT:}

This is an Accepted Manuscript of an article published by Taylor \& Francis in 'Optimization Methods and Software' on 23 Mar 2020 (published online), available online: https://www.tandfonline.com/10.1080/10556788.2020.1740219; The fulltext file will be made open to the public on 23 Mar 2021 in accordance with publisher's 'Terms and Conditions for SelfArchiving'; This is not the published version. Please cite only the published version. この論文は出版社版でありません。

引用の際には出版社版をご確認ご利用ください 


\title{
Signed Ring Families and Signed Posets*
}

\author{
Kazutoshi Ando ${ }^{\dagger}$ and Satoru Fujishige ${ }^{\ddagger}$
}

ARTICLE HISTORY

Compiled December 26, 2019

\begin{abstract}
The one-to-one correspondence between finite distributive lattices and finite partially ordered sets (posets) is a well-known theorem of G. Birkhoff. This implies a nice representation of any distributive lattice by its corresponding poset, where the size of the former (distributive lattice) is often exponential in the size of the underlying set of the latter (poset). A lot of engineering and economic applications bring us distributive lattices as a ring family of sets which is closed with respect to the set union and intersection. When it comes to a ring family of sets, the underlying set is partitioned into subsets (or components) and we have a poset structure on the partition. This is a set-theoretical variant of the Birkhoff theorem revealing the correspondence between finite ring families and finite posets on partitions of the underlying sets, which was pursued by Masao Iri around 1978, especially concerned with what is called the principal partition of discrete systems such as graphs, matroids, and polymatroids.

In the present paper we investigate a signed-set version of the Birkhoff-Iri decomposition in terms of signed ring family, which corresponds to Reiner's result on signed posets, a signed counterpart of the Birkhoff theorem. We show that given a signed ring family, we have a signed partition of the underlying set together with a signed poset on the signed partition which represents the given signed ring family. This representation is unique up to certain reflections.
\end{abstract}

\section{KEYWORDS}

Signed ring families; signed posets; bidirected graphs; decomposition; bisubmodular functions

\section{Introduction}

The one-to-one correspondence between finite distributive lattices and finite partially ordered sets (posets) is a well-known theorem of G. Birkhoff (see [6,7]). This implies a nice representation of any distributive lattice by its corresponding poset, where the size of the former (distributive lattice) is often exponential in the size of the underlying set of the latter (poset). A lot of engineering and economic applications bring us distributive lattices as a ring family of sets which is closed with respect to the two binary operations of set union and intersection. Typically we have such a ring family of sets as a family of minimizers of a submodular set

* The original version of the present paper is a technical report [1], which has been left unpublished while its copy being often requested by interested researchers. We have now decided to try to make it published by submitting it to the present special issue in memory of Masao Iri, after substantial revisions made by clarifying a relationship between some results of Iri and ours, by improving the description of our main contribution, and by including some recent related references.

$\dagger$ Faculty of Engineering, Shizuoka University, Hamamatsu, Shizuoka 432-8561, Japan. Email: ando.kazutoshi@shizuoka.ac.jp

$\ddagger$ CONTACT S. Fujishige. Research Institute for Mathematical Sciences, Kyoto University, Kyoto 606-8502, Japan. Email: fujishig@kurims.kyoto-u.ac.jp 
function (see [13]). When it comes to a ring family of sets, the underlying set is partitioned into subsets (or components) and we have a poset structure on the partition. This decomposition with a poset structure on the set of components plays important and crucial roles in many practical problems related, for example, to the decomposition of a directed graph into strongly connected components, the Dulmage-Mendelsohn decomposition of a bipartite graph ([29]), etc. This is a set-theoretical variant of the original Birkhoff theorem, to be called the Birkhoff-Iri decomposition, revealing the correspondence between finite ring families and finite posets on partitions of the underlying sets, which was intensively pursued by Masao Iri around 1978, especially concerned with the problem of what is called the principal partition of discrete systems $([14,22-25,28])$.

Theorem 1.1 (Birkhoff-Iri). Let $\mathcal{D}$ be a set of subsets of a finite set $V$ with $\emptyset, V \in \mathcal{D}$. Then $\mathcal{D}$ is a distributive lattice with set union and intersection as the lattice operations if and only if there exists a poset $(\Pi(V), \preceq)$ on a partition $\Pi(V)$ of $V$ such that $\mathcal{D}$ is expressed as follows:

$$
X \in \mathcal{D} \Longleftrightarrow \text { there exists an ideal } \mathcal{J} \text { of }(\Pi(V), \preceq) \text { such that } X=\bigcup_{F \in \mathcal{J}} F,
$$

where $\mathcal{J}$ is an ideal of $(\Pi(V), \preceq)$ if for every $F \in \mathcal{J}$ we have $F^{\prime} \in \mathcal{J}$ for all $F^{\prime} \in \Pi(V)$ satisfying $F^{\prime} \preceq F$.

Example 1: See Figure 1 as an illustration of the Birkhoff-Iri decomposition of the underlying set with the associated poset structure on the partition.

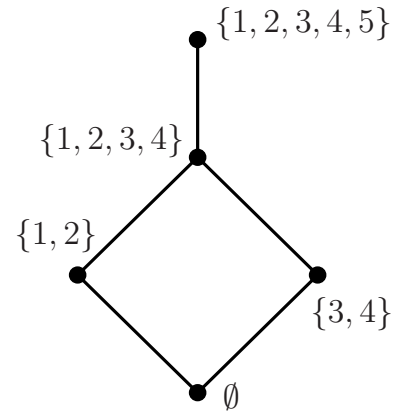

(a)

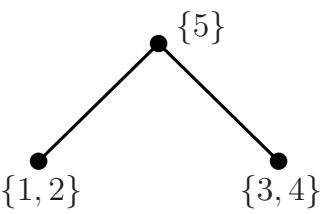

(b)

Figure 1. (a) A distributive set lattice $\mathcal{D}$ and (b) its corresponding poset on the partition of the underlying set $\{1,2,3,4,5\}$.

On the other hand, V. Reiner [32] introduced the concept of signed poset and showed the so-called signed Birkhoff theorem, which is a signed analogue of the Birkhoff theorem on distributive lattices and posets. The signed Birkhoff theorem [32, Theorem 4.8] asserts that for a finite lattice $\mathcal{L}$ with the maximum element $\hat{1}, \mathcal{L}-\{\hat{1}\}$ is isomorphic to the set of ideals of some signed poset $\mathcal{P}$ if and only if $\mathcal{L}$ is $B_{n}$-distributive, where $\mathcal{P}$ is determined by $\mathcal{L}$ up to isomorphism as a signed poset (see [32] for the precise definitions and terminology).

In the present paper we investigate a signed-set version of the Birkhoff-Iri decomposition (Theorem 1.1), which gives a signed-set decomposition of discrete systems represented by signed ring families into signed partitions with signed poset structures (the precise description will be given in Sections 4 and 5). This is an elaboration of Reiner's result on $B_{n}$-distributive lattices and signed posets to obtain a signed-set counterpart of the Birkhoff-Iri theorem (Theorem 1.1), in terms of signed ring family.

The present paper is organized as follows. Basic definitions and preliminaries are given 
in Sections 2 and 3. Section 4 describes how every simple and spanning signed ring family can be represented by a signed poset introduced by Reiner, or equivalently by a bidirected graph considered in Section 3, in terms of signed ring family (see Theorem 4.10). We further consider general signed ring families and their representation by signed partitions with signed poset structures in Section 5 (see Theorem 5.1). Finally, in Section 6 we give some remarks on recent related developments in signed ring families and bisubmodularity.

\section{Preliminaries}

The authors ([2-4]) in collaboration with T. Naitoh and T. Nemoto were interested in a family $\mathcal{F} \subseteq 3^{V} \equiv\{(X, Y) \mid X, Y \subseteq V, X \cap Y=\emptyset\}$ for a finite nonempty set $V$ that is closed with respect to the two binary operations called reduced union $\sqcup$ and intersection $\sqcap$. For each $\left(X_{i}, Y_{i}\right) \in \mathcal{F}(i=1,2)$ the reduced union $\left(X_{1}, Y_{1}\right) \sqcup\left(X_{2}, Y_{2}\right)$ and the intersection $\left(X_{1}, Y_{1}\right) \sqcap\left(X_{2}, Y_{2}\right)$ are, respectively, defined by

$$
\begin{aligned}
& \left(X_{1}, Y_{1}\right) \sqcup\left(X_{2}, Y_{2}\right)=\left(\left(X_{1} \cup X_{2}\right) \backslash\left(Y_{1} \cup Y_{2}\right),\left(Y_{1} \cup Y_{2}\right) \backslash\left(X_{1} \cup X_{2}\right)\right), \\
& \left(X_{1}, Y_{1}\right) \sqcap\left(X_{2}, Y_{2}\right)=\left(X_{1} \cap X_{2}, Y_{1} \cap Y_{2}\right) .
\end{aligned}
$$

(See Figure 2.)

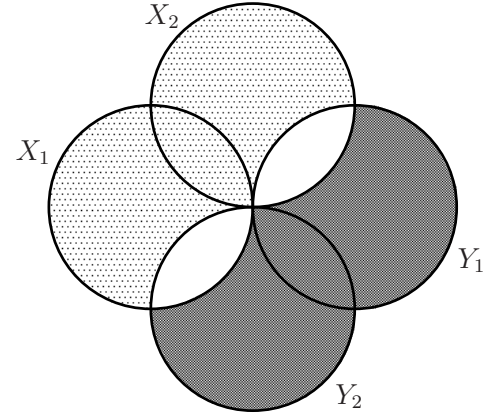

(a)

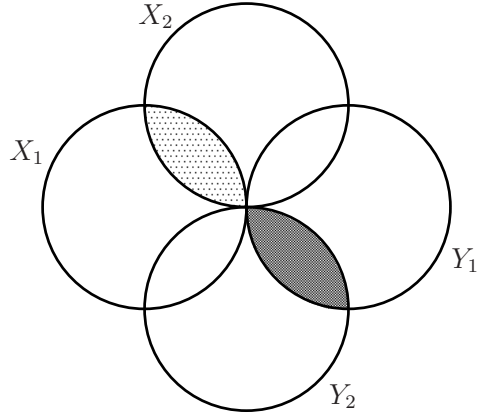

(b)

Figure 2. (a) The reduced union $\left(X_{1}, Y_{1}\right) \sqcup\left(X_{2}, Y_{2}\right)$ and (b) the intersection $\left(X_{1}, Y_{1}\right) \sqcap\left(X_{2}, Y_{2}\right)$.

Such a family $\mathcal{F}$ is called $\sqcup, \sqcap$-closed. We call $\mathcal{F}$ a $\sqcup, \sqcap$-closed family or a signed ring family (the former term was used in our original report [1] but we use the latter in the following).

Each $(X, Y) \in \mathcal{F}$ can be identified with its characteristic vector $\chi_{(X, Y)} \in\{0, \pm 1\}^{V}$ defined by

$$
\chi_{(X, Y)}(v)=\left\{\begin{aligned}
1 & \text { if } v \in X \\
-1 & \text { if } v \in Y \\
0 & \text { otherwise }
\end{aligned}\right.
$$

for each $v \in V$. Therefore, we call each $(X, Y) \in \mathcal{F}$ a signed set. Also, we call $(\emptyset, \emptyset)$ the null signed set. It should be noted that we have the following equality for all $\left(X_{i}, Y_{i}\right) \in \mathcal{F}$ $(i=1,2)$.

$$
\chi_{\left(X_{1}, Y_{1}\right)}+\chi_{\left(X_{2}, Y_{2}\right)}=\chi_{\left(X_{1}, Y_{1}\right) \sqcup\left(X_{2}, Y_{2}\right)}+\chi_{\left(X_{1}, Y_{1}\right) \sqcap\left(X_{2}, Y_{2}\right)} .
$$


For $\left(X_{i}, Y_{i}\right) \in \mathcal{F}(i=1,2)$ we write $\left(X_{1}, Y_{1}\right) \sqsubseteq\left(X_{2}, Y_{2}\right)$ if $X_{1} \subseteq X_{2}$ and $Y_{1} \subseteq Y_{2}$. If we have $\left(X_{1}, Y_{1}\right) \sqsubseteq\left(X_{2}, Y_{2}\right)$ and $\left(X_{1}, Y_{1}\right) \neq\left(X_{2}, Y_{2}\right)$, we write $\left(X_{1}, Y_{1}\right) \sqsubset\left(X_{2}, Y_{2}\right)$. See Figure 3 for an example of a signed ring family on $V=\{p, q, r\}$.

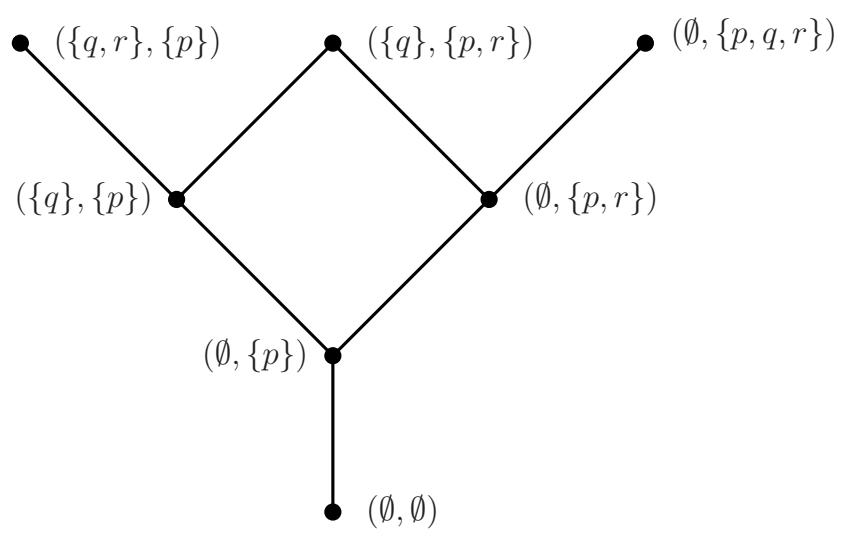

Figure 3. A signed ring family where the signed sets are ordered by $\sqsubseteq$.

A special signed ring family $\mathcal{F}=3^{V} \equiv\{(X, Y) \mid X, Y \subseteq V, X \cap Y=\emptyset\}$ appears in $[8-10,13,31]$, while signed ring families were introduced as domains of bisubmodular functions (see $[3,13]$ ). A bisubmodular function $f: \mathcal{F} \rightarrow \mathbb{R}$ is a function satisfying

$$
f\left(X_{1}, Y_{1}\right)+f\left(X_{2}, Y_{2}\right) \geq f\left(\left(X_{1}, Y_{1}\right) \sqcup\left(X_{2}, Y_{2}\right)\right)+f\left(\left(X_{1}, Y_{1}\right) \sqcap\left(X_{2}, Y_{2}\right)\right)
$$

for all $\left(X_{i}, Y_{i}\right) \in \mathcal{F}(i=1,2)$. Bisubmodular functions are generalization of rank functions of polymatroids and other related polyhedra (see, e.g., $[8-10,13,31])$. Given a bisubmodular function $f: \mathcal{F} \rightarrow \mathbb{R}$ on a signed ring family $\mathcal{F}$ with $(\emptyset, \emptyset) \in \mathcal{F}$ and $f(\emptyset, \emptyset)=0$, we define the bisubmodular polyhedron associated with $f$ by

$$
\mathrm{P}_{*}(f)=\left\{x \mid x \in \mathbb{R}^{V}, \forall(X, Y) \in \mathcal{F}: x(X, Y) \leq f(X, Y)\right\},
$$

where $x(X, Y)=\sum_{v \in X} x(v)-\sum_{v \in Y} x(v)$. The class of bisubmodular polyhedra is exactly the one for which the greedy algorithm works (see $[9,10]$ for $\mathcal{F}=3^{V}$ ). Real-valued functions defined on $3^{V}$ are also studied in the field of game theory under the name of bicooperative games or bicapacities where the partial order on $3^{V}$ is different from that considered in this paper (see, e.g., $[5,11,20,21])$.

We say $\mathcal{F}$ is spanning if there exists some $(X, Y) \in \mathcal{F}$ such that $X \cup Y=V$. We can show that a signed ring family $\mathcal{F}$ is spanning if and only if for each $v \in V$ there exists some $(X, Y) \in \mathcal{F}$ such that $v \in X \cup Y$. This follows from the following lemma.

Lemma 2.1. Every maximal element $(X, Y) \in \mathcal{F}$ has the same set $X \cup Y$, where the order among $\mathcal{F}$ is with respect to $\sqsubseteq$.

(Proof) Since

$$
\left(X_{1} \cup\left(X_{2} \backslash Y_{1}\right), Y_{1} \cup\left(Y_{2} \backslash X_{1}\right)\right)=\left(\left(X_{1}, Y_{1}\right) \sqcup\left(X_{2}, Y_{2}\right)\right) \sqcup\left(X_{1}, Y_{1}\right),
$$

we have

$$
\left(X_{1} \cup\left(X_{2} \backslash Y_{1}\right), Y_{1} \cup\left(Y_{2} \backslash X_{1}\right)\right) \in \mathcal{F}
$$


for any $\left(X_{i}, Y_{i}\right) \in \mathcal{F}(i=1,2)$. Also, note that we have

$$
\begin{gathered}
\left(X_{1}, Y_{1}\right) \sqsubseteq\left(X_{1} \cup\left(X_{2} \backslash Y_{1}\right), Y_{1} \cup\left(Y_{2} \backslash X_{1}\right)\right), \\
X_{1} \cup\left(X_{2} \backslash Y_{1}\right) \cup Y_{1} \cup\left(Y_{2} \backslash X_{1}\right)=X_{1} \cup X_{2} \cup Y_{1} \cup Y_{2} .
\end{gathered}
$$

Therefore, the present lemma follows from (7) (10).

Due to Lemma 2.1, for any maximal $(X, Y) \in \mathcal{F}$ let us call the unique set $X \cup Y$ the support of $\mathcal{F}$. We thus see that for a non-spanning signed ring family $\mathcal{F} \subseteq 3^{V}$ on $V$ we can restrict the underlying set $V$ to its support. Therefore, without loss of generality we assume that $\mathcal{F}$ is spanning in the sequel unless otherwise stated.

For a spanning $\mathcal{F}$ we say $\mathcal{F}$ is simple if for each distinct $v, w \in V$

- there exists some $(X, Y) \in \mathcal{F}$ such that either $v \in X \cup Y$ and $w \notin X \cup Y$ or $v \notin X \cup Y$ and $w \in X \cup Y$.

We shall consider representations of general non-simple signed ring families by means of signed posets. Before doing so, we first treat simple signed ring families. We show a theorem (Theorem 4.10) that there exists a one-to-one correspondence between the set of all the simple signed ring families $\mathcal{F} \subseteq 3^{V}$ on $V$ with $(\emptyset, \emptyset) \in \mathcal{F}$ and the set of all the signed posets $\mathcal{P}$ on $V$ such that each such $\mathcal{F}$ is the set of all the ideals of the corresponding signed poset $\mathcal{P}$. The theorem, Theorem 4.10, is a set-theoretical version of the signed Birkhoff theorem of V. Reiner [32], which sheds a new light on the signed Birkhoff theorem. We further examine the representations of general non-simple and/or non-spanning signed ring families later in Section 5 .

The result obtained in this paper gives an important basis for developing a theory of bisubmodular functions and associated (possibly unbounded) bisubmodular polyhedra. It should be noted that the collection of all the minimizers of an arbitrary bisubmodular function is a signed ring family. Hence, for any point $x$ in the bisubmodular polyhedron defined by (6) the collection $\mathcal{F}(x)$ of tight signed sets given by

$$
\mathcal{F}(x)=\{(X, Y) \mid(X, Y) \in \mathcal{F}, x(X, Y)=f(X, Y)\}
$$

forms a signed ring subfamily of $\mathcal{F}$. We can see that a point $x \in \mathrm{P}_{*}(f)$ is an extreme point of $\mathrm{P}_{*}(f)$ if and only if $\mathcal{F}(x)$ is simple (see [2]). Therefore, for each extreme point $x$ of the bisubmodular polyhedron $\mathrm{P}_{*}(f)$ we have a signed poset associated with $\mathcal{F}(x)$. We can characterize the adjacency of extreme points of the bisubmodular polyhedron in terms of the Hasse diagrams of the associated signed posets. By means of the signed poset representation we can further examine facets, faces, dimensions, connected components, the membership problem etc. for bisubmodular polyhedra (see [2]). Further related topics and recent developments will be seen in Section 6.

\section{Signed Posets and Ideals}

Following Reiner [32], we define a signed poset and its ideal in an equivalent but slightly different way in terms of bidirected graph.

For a finite vertex set $V$ and a finite arc set $A$ we are given a boundary operator $\partial$ on $A$ as follows. For each arc $a \in A$ there exist some vertices $v, w \in V$ such that one of the following three holds:

(1) $\partial a=v+w \quad(\operatorname{arc} a$ has two tails, one at $v$ and one at $w)$, 
(2) $\partial a=-v-w \quad(\operatorname{arc} a$ has two heads, one at $v$ and one at $w)$,

(3) $\partial a=v-w \quad(\operatorname{arc} a$ has a tail at $v$ and a head at $w)$,

where the right-hand sides should be regarded as elements of the free module over the set $\mathbb{Z}$ of integers with a base $V$, and if $v=w$, we do not allow (3). Also, we assume that there do not exist any two distinct $\operatorname{arcs} a, a^{\prime} \in A$ such that $\partial a=\partial a^{\prime}$. When $\partial a= \pm v \pm w$, we say arc $a$ is incident to $v$ (and $w$ ) and it is sometimes convenient to write $\operatorname{arc} a$ as $v \pm \pm w$. If arc $a$ is incident to only one vertex $v$ (i.e., $a=v++v$ or $a=v--v$ ), arc $a$ is called a selfloop at $v$. We call $G=(V, A ; \partial)$ a bidirected graph. See Figure 4 for an example of a bidirected graph.

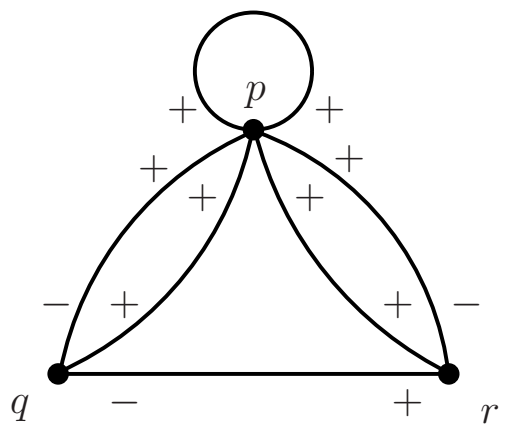

Figure 4. A bidirected graph.

For a bidirected graph $G=(V, A ; \partial)$ we further assume the following:

(i) There are no two $\operatorname{arcs} a_{1}, a_{2} \in A$ such that $\partial a_{1}=-\partial a_{2}$ (i.e., no two arcs, of either $u++v$ and $u--v$ or $u+-v$ and $u-+v$, coexist).

(ii) For any two arcs $a_{1}, a_{2} \in A$, if the sum of the absolute values of the coefficients of the vertices in $\partial a_{1}+\partial a_{2}$ is two, then there exists an arc $a_{3} \in A$ such that $\partial a_{3}=\partial a_{1}+\partial a_{2}$ (i.e., the existence of arcs $u+ \pm v$ and $v \mp+w$ implies the existence of arc $u++w$ and similarly for other sign patterns).

(iii) For any two selfloops $a_{1}, a_{2} \in A$ incident to distinct vertices (e.g., $a_{1}=u++u$ and $a_{2}=v++v$ for $u \neq v$ ) there exists an arc $a_{3} \in A$ such that $2 \partial a_{3}=\partial a_{1}+\partial a_{2}$ (e.g., $\left.a_{3}=u++v\right)$.

Then, we call the bidirected graph $G=(V, A ; \partial)$ a signed poset on $V$ and denote it by $\mathcal{P}=(V, A ; \partial)$ as well. This definition is equivalent to the one given by Reiner [32]. A signed poset $\mathcal{P}=(V, A ; \partial)$ on $V$ is uniquely determined by $\partial A \equiv\{\partial a \mid a \in A\}$, i.e., a signed poset is uniquely determined up to the relabeling of the arcs. We can see that the bidirected graph shown in Figure 4 is in fact a signed poset.

For each $a \in A$ we denote by $\partial^{+} a\left(\partial^{-} a\right)$ the set of the vertices that have positive (negative) coefficients in $\partial a$.

Now, we define an ideal of the signed poset $\mathcal{P}=(V, A ; \partial)$ as follows. A signed set $(X, Y) \in 3^{V}$ is an ideal of $\mathcal{P}$ if it satisfies

$$
\langle\partial a,(X, Y)\rangle \leq 0 \quad(a \in A),
$$

where $\partial a$ and $(X, Y)$ should be regarded as integral vectors in $\mathbb{Z}^{V}$ in a natural way, and $\langle\cdot, \cdot\rangle$ as the canonical inner product. Inequality (12) means that $\left(\partial^{+} a \cap X\right) \cup\left(\partial^{-} a \cap Y\right) \neq \emptyset$ implies $\left(\partial^{-} a \cap X\right) \cup\left(\partial^{+} a \cap Y\right) \neq \emptyset$. In Reiner's definition of ideals the inequality sign in (12) is reversed but we adopt the above definition due only to the consistency with our system of 
notations for ordinary posets and ideals (cf. [13]). For the signed poset shown in Figure 4, the set of the ideals is exactly the signed ring family given in Figure 3.

\section{Signed Ring Families and Their Representations}

Let $\mathcal{F} \subseteq 3^{V}$ be a simple signed ring family with $(\emptyset, \emptyset) \in \mathcal{F}$. For each $v \in V$ define

$$
\mathrm{F}(+v)=\sqcap\{(X, Y) \mid v \in X,(X, Y) \in \mathcal{F}\}
$$

if there exists some $(X, Y) \in \mathcal{F}$ such that $v \in X$, and define

$$
\mathrm{F}(-v)=\sqcap\{(X, Y) \mid v \in Y,(X, Y) \in \mathcal{F}\}
$$

if there exists some $(X, Y) \in \mathcal{F}$ such that $v \in Y$. If there is no $(X, Y) \in \mathcal{F}$ such that $v \in X$ (or $v \in Y$ ), then we define $\mathrm{F}(+v)=(\emptyset, \emptyset)$ (or $\mathrm{F}(-v)=(\emptyset, \emptyset)$ ). Note that since $\mathcal{F}$ is a spanning family on $V$, by Lemma $2.1 \mathrm{~F}(+v)$ or $\mathrm{F}(-v)$ is non-null for every $v \in V$. Also, for any $W=(X, Y) \in \mathcal{F}$ we define

$$
W^{+}=X, \quad W^{-}=Y .
$$

Given a simple (and hence spanning) signed ring family $\mathcal{F} \subseteq 3^{V}$ on $V$, we construct a bidirected graph $G(\mathcal{F})$ as follows. $G(\mathcal{F})$ has the vertex set $V$ (since $\mathcal{F}$ is spanning). The arc set $A$ is constructed by the following procedures (1) (3):

(1) For each $v \in V$,

(1a) if $\mathrm{F}(-v)=(\emptyset, \emptyset)$, add a selfloop $a$ at $v$ such that $\partial a=-2 v$ (i.e., $a=v--v$ ),

(1b) if $\mathrm{F}(+v)=(\emptyset, \emptyset)$, add a selfloop $a$ at $v$ such that $\partial a=2 v$ (i.e., $a=v++v$ ).

(2) For each distinct $v, w \in V$,

(2a) if $w \in \mathrm{F}(+v)^{+}$, add an arc $a$ such that $\partial a=v-w$ (i.e., $a=v+-w$ ),

(2b) if $w \in \mathrm{F}(+v)^{-}$, add an arc $a$ such that $\partial a=v+w$ (i.e., $a=v++w$ ),

(2c) if $w \in \mathrm{F}(-v)^{+}$, add an arc $a$ such that $\partial a=-v-w$ (i.e., $a=v--w$ ),

(2d) if $w \in \mathrm{F}(-v)^{-}$, add an arc $a$ such that $\partial a=-v+w$ (i.e., $a=v-+w$ ).

(3) For any two selfloops $a_{1}$ and $a_{2}$ that are incident to distinct vertices, add an arc $a_{3}$ such that $2 \partial a_{3}=\partial a_{1}+\partial a_{2}$.

During the construction of the arc set $A$, if an arc to be added has already been constructed, then we skip the operation. It should be noted that if we do not require condition (iii) for the signed poset, or more precisely if we remove such an arc $a_{3}$ appearing in condition (iii), then we do not need Procedure (3) given above.

Let us consider the signed ring family $\mathcal{F}$ shown in Figure 3. We see the following.

$$
\begin{array}{ll}
\mathrm{F}(+p)=(\emptyset, \emptyset), & \mathrm{F}(-p)=(\emptyset,\{p\}), \\
\mathrm{F}(+q)=(\{q\},\{p\}), & \mathrm{F}(-q)=(\emptyset,\{p, q, r\}), \\
\mathrm{F}(+r)=(\{q, r\},\{p\}), & \mathrm{F}(-r)=(\emptyset,\{p, r\}) .
\end{array}
$$

Then, procedures (1) (3) yield the bidirected graph $G(\mathcal{F})$ in Figure 4.

To show that the bidirected graph $G(\mathcal{F})$ constructed above is a signed poset, we need some lemmas.

Lemma 4.1. For any distinct $v, w \in V$, 
(a) if $w \in \mathrm{F}(+v)^{+}$, then $v \notin \mathrm{F}(+w)^{+}$,

(b) if $w \in \mathrm{F}(+v)^{-}$, then $v \notin \mathrm{F}(-w)^{+}$,

(c) if $w \in \mathrm{F}(-v)^{+}$, then $v \notin \mathrm{F}(+w)^{-}$,

(d) if $w \in \mathrm{F}(-v)^{-}$, then $v \notin \mathrm{F}(-w)^{-}$.

(Proof) We show (a) (the proofs of the other cases are similar).

Suppose, to the contrary, that $w \in \mathrm{F}(+v)^{+}$and $v \in \mathrm{F}(+w)^{+}$. Since $\mathcal{F}$ is simple, there is some $(X, Y) \in \mathcal{F}$ such that (1) $v \in X \cup Y$ and $w \notin X \cup Y$, or (2) $v \notin X \cup Y$ and $w \in X \cup Y$. In Case (1), if $v \in X$, then $(\mathrm{F}(+v) \sqcap(X, Y))^{+}$contains $v$ but not $w$, which contradicts the minimality of $\mathrm{F}(+v)$; and if $v \in Y$, then $(\mathrm{F}(+w) \sqcup(X, Y))^{+}$contains $w$ but not $v$, which contradicts the minimality of $\mathrm{F}(+w)$. Case (2) can be treated similarly as Case (1).

Lemma 4.2. For any distinct $v, w \in V$,

(a) if $w \in \mathrm{F}(+v)^{+}$, then $\mathrm{F}(+w) \sqsubset \mathrm{F}(+v)$,

(b) if $w \in \mathrm{F}(+v)^{-}$, then $\mathrm{F}(-w) \sqsubset \mathrm{F}(+v)$,

(c) if $w \in \mathrm{F}(-v)^{+}$, then $\mathrm{F}(+w) \sqsubset \mathrm{F}(-v)$,

(d) if $w \in \mathrm{F}(-v)^{-}$, then $\mathrm{F}(-w) \sqsubset \mathrm{F}(-v)$.

(Proof) If the inclusion $\sqsubset$ is replaced by the inclusion $\sqsubseteq$ with equality, then each assertion easily follows from the definition (the minimality) of $\mathrm{F}(\cdot)$. The strict inclusion $\sqsubset$ is due to Lemma 4.1.

Lemma 4.3. For any distinct $v, w \in V$,

(a) if $w \in \mathrm{F}(+v)^{+}$and $\mathrm{F}(-w) \neq(\emptyset, \emptyset)$, then $v \in \mathrm{F}(-w)^{-}$,

(b) if $w \in \mathrm{F}(+v)^{-}$and $\mathrm{F}(+w) \neq(\emptyset, \emptyset)$, then $v \in \mathrm{F}(+w)^{-}$,

(c) if $w \in \mathrm{F}(-v)^{+}$and $\mathrm{F}(-w) \neq(\emptyset, \emptyset)$, then $v \in \mathrm{F}(-w)^{+}$,

(d) if $w \in \mathrm{F}(-v)^{-}$and $\mathrm{F}(+w) \neq(\emptyset, \emptyset)$, then $v \in \mathrm{F}(+w)^{+}$.

(Proof) We show (a) (the proof of the other cases are similar).

Suppose, to the contrary, that $w \in \mathrm{F}(+v)^{+}, \mathrm{F}(-w) \neq(\emptyset, \emptyset)$ and $v \notin \mathrm{F}(-w)^{-}$. Then we have $v \in(\mathrm{F}(+v) \sqcup \mathrm{F}(-w))^{+}$and $w \notin(\mathrm{F}(+v) \sqcup \mathrm{F}(-w))^{+}$, which contradicts the minimality of $\mathrm{F}(+v)$.

Lemma 4.3 partly corresponds to Proposition 4.6 in [32].

Lemma 4.4. For any distinct $v, w \in V$,

(a) if $w \in \mathrm{F}(+v)^{+}$and $\mathrm{F}(-v)=(\emptyset, \emptyset)$, then $\mathrm{F}(-w)=(\emptyset, \emptyset)$,

(b) if $w \in \mathrm{F}(+v)^{-}$and $\mathrm{F}(-v)=(\emptyset, \emptyset)$, then $\mathrm{F}(+w)=(\emptyset, \emptyset)$,

(c) if $w \in \mathrm{F}(-v)^{+}$and $\mathrm{F}(+v)=(\emptyset, \emptyset)$, then $\mathrm{F}(-w)=(\emptyset, \emptyset)$,

(d) if $w \in \mathrm{F}(-v)^{-}$and $\mathrm{F}(+v)=(\emptyset, \emptyset)$, then $\mathrm{F}(+w)=(\emptyset, \emptyset)$.

(Proof) We show (a) (the proof of the other cases are similar).

Suppose, to the contrary, that $w \in \mathrm{F}(+v)^{+}, \mathrm{F}(-v)=(\emptyset, \emptyset)$ and $\mathrm{F}(-w) \neq(\emptyset, \emptyset)$. Then from Lemma 4.3 we have $v \in \mathrm{F}(-w)^{-}$, which contradicts the assumption that $\mathrm{F}(-v)=$ $(\emptyset, \emptyset)$.

From Lemmas 4.1 4.4 we have the following.

Theorem 4.5. The bidirected graph $G(\mathcal{F})=(V, A ; \partial)$ defined above is a signed poset.

(Proof) Let us check conditions (i) (iii) in the definition of a signed poset.

(i) Suppose, to the contrary, that there exist two arcs $a_{1}, a_{2} \in A$ such that $\partial a_{1}+\partial a_{2}=0$. 
These $a_{1}$ and $a_{2}$ are both non-selfloop arcs or both selfloop arcs. Since selfloop arcs are added only by Procedure (1), the case of two selfloops is excluded due to the remark given after the definition of $\mathrm{F}(\cdot)$. Hence, suppose $a_{1}$ and $a_{2}$ are non-selfloop arcs. By the same reason as above, they are not both added by Procedure (3). Suppose that $a_{1}$ and $a_{2}$ are both added by Procedure (2). We can assume without loss of generality that $\partial a_{1}=v+w$ and $\partial a_{2}=-v-w$ since the other cases are treated similarly. Then we have one of the following (a) (d):
(a) $w \in \mathrm{F}(+v)^{-}$and $v \in \mathrm{F}(-w)^{+}$;
(b) $w \in \mathrm{F}(+v)^{-}$and $w \in \mathrm{F}(-v)^{+}$;
(c) $v \in \mathrm{F}(+w)^{-}$and $v \in \mathrm{F}(-w)^{+}$;
(d) $v \in \mathrm{F}(+w)^{-}$and $w \in \mathrm{F}(-v)^{+}$.

Cases (a) and (d) are excluded due to Lemma 4.1. Case (b) is reduced to Case (a) since $w \in \mathrm{F}(+v)^{-}$and $w \in \mathrm{F}(-v)^{+}$imply $v \in \mathrm{F}(-w)^{+}$by Lemma 4.3. Similarly, Case (c) is reduced to Case (d). The only possibility is now the case when one is added by Procedure (2) and the other by Procedure (3). This case is also excluded by Lemma 4.4.

(ii) Suppose $\partial a_{1}+\partial a_{2}= \pm v \pm w$ for some $v, w \in V$. We treat only the case when $\partial a_{1}+\partial a_{2}=v+w$ since the other cases are treated similarly. Then we have either $v-u \in \partial A$ and $u+w \in \partial A$ or $u+v \in \partial A$ and $w-u \in \partial A$. We assume the former case $(v-u \in \partial A$ and $u+w \in \partial A$ ) without loss of generality.

When $u=w$, we have $\mathrm{F}(+w)=(\emptyset, \emptyset)$ and hence $\mathrm{F}(-w) \neq(\emptyset, \emptyset)$. Also, since $v-w \in$ $\partial A$, we have either $w \in \mathrm{F}(+v)^{+}$or $v \in \mathrm{F}(-w)^{-}$. The former case reduces to the latter by Lemma 4.3. Then, by Lemma 4.4, we have $\mathrm{F}(+v)=(\emptyset, \emptyset)$, which implies $2 v \in \partial A$. Hence, Procedure (3) constructs an arc $a_{3} \in A$ with $\partial a_{3}=v+w$.

When $u \neq w$ and $v \neq w$, we have one of the following (a) (f):

(a) $v \in \mathrm{F}(-u)^{-}, u \in \mathrm{F}(+w)^{-}$;

(b) $u \in \mathrm{F}(+v)^{+}, w \in \mathrm{F}(+u)^{-}$;

(c) $v \in \mathrm{F}(-u)^{-}, w \in \mathrm{F}(+u)^{-}$;

(d) $u \in \mathrm{F}(+v)^{+}, u \in \mathrm{F}(+w)^{-}$;

(e) $\mathrm{F}(+v)=(\emptyset, \emptyset), \mathrm{F}(-u)=(\emptyset, \emptyset), w \in \mathrm{F}(+u)^{-}$;

(f) $\mathrm{F}(+w)=(\emptyset, \emptyset), \mathrm{F}(+u)=(\emptyset, \emptyset), v \in \mathrm{F}(-u)^{-}$.

Both (a) and (b) imply $v+w \in \partial A$ from Lemma 4.2. In Case (c), if both $\mathrm{F}(+v)=(\emptyset, \emptyset)$ and $\mathrm{F}(+w)=(\emptyset, \emptyset)$, then we have $v+w \in \partial A$ due to Procedure (3). Moreover, if $\mathrm{F}(+w) \neq(\emptyset, \emptyset)$ (or $\mathrm{F}(+v) \neq(\emptyset, \emptyset)$ ), then Case (c) is reduced to Case (a) (or Case (b)) from Lemma 4.3. Case (d) is reduced to Case (a) (and Case (b)) from Lemma 4.3 since $\mathrm{F}(-u) \neq(\emptyset, \emptyset)$ (and $\mathrm{F}(+u) \neq(\emptyset, \emptyset)$ ). In Case (e) (or Case (f)) we have $\mathrm{F}(+w)=(\emptyset, \emptyset)$ (or $\mathrm{F}(+v)=(\emptyset, \emptyset)$ ) due to Lemma 4.4, so that we have $v+w \in \partial A$ by Procedure (3).

Let us consider the case when $u \neq w$ and $v=w$. If $\mathrm{F}(+v) \neq(\emptyset, \emptyset)$, then $\operatorname{arcs} a_{1}$ and $a_{2}$ with $\partial a_{1}=v-u$ and $\partial a_{2}=v+u$ are constructed by Procedure (2). Then we have (I) $v \in \mathrm{F}(+u)^{-}$or $u \in \mathrm{F}(+v)^{-}$and (II) $v \in \mathrm{F}(-u)^{-}$or $u \in \mathrm{F}(+v)^{+}$. However, by Lemma 4.3, (I) implies $u \in \mathrm{F}(+v)^{-}$and (II) implies $u \in \mathrm{F}(+v)^{+}$, a contradiction. Therefore, we have $\mathrm{F}(+v)=(\emptyset, \emptyset)$, and hence, by Procedure (3) there is a selfloop $a$ with $\partial a=2 v$.

(iii) This is due to the definition of $G(\mathcal{F})$.

We now denote the signed poset $G(\mathcal{F})$ by $\mathcal{P}(\mathcal{F})=(V, A ; \partial)$.

Lemma 4.6. Let $(X, Y) \in 3^{V}$ be an ideal of the signed poset $\mathcal{P}(\mathcal{F})$. Then we have

$$
\begin{array}{ll}
(\emptyset, \emptyset) \neq \mathrm{F}(+v) \sqsubseteq(X, Y) & (v \in X), \\
(\emptyset, \emptyset) \neq \mathrm{F}(-v) \sqsubseteq(X, Y) \quad(v \in Y) .
\end{array}
$$

(Proof) Since $(X, Y)$ is an ideal of $\mathcal{P}(\mathcal{F})$, relations (16) and (17) follow from the definition 
of $\mathcal{P}(\mathcal{F})$.

Now, we show the following theorem.

Theorem 4.7. The set of all the ideals of the signed poset $\mathcal{P}(\mathcal{F})$ coincides with the given $\mathcal{F}$.

(Proof) Suppose that $(X, Y) \in 3^{V}$ is an ideal of $\mathcal{P}(\mathcal{F})$. From Lemma 4.6 we have

$$
(X, Y)=\left(\sqcup_{v \in X} \mathrm{~F}(+v)\right) \sqcup\left(\sqcup_{v \in Y} \mathrm{~F}(-v)\right) .
$$

Hence, $(X, Y) \in \mathcal{F}$.

Conversely, suppose $(X, Y) \in \mathcal{F}$. Then we have

$$
\begin{array}{ll}
(\emptyset, \emptyset) \neq \mathrm{F}(+v) \sqsubseteq(X, Y) & (v \in X), \\
(\emptyset, \emptyset) \neq \mathrm{F}(-v) \sqsubseteq(X, Y) \quad(v \in Y) .
\end{array}
$$

If $(X, Y)$ is not an ideal of $\mathcal{P}(\mathcal{F})$, then we have the following (I) or (II):

(I) For some $v \in X$ we have one of the following three:

(a) There is a selfloop $a$ such that $\partial a=2 v$.

(b) There are a non-selfloop arc $a$ and a vertex $w \notin Y$ such that $\partial a=v+w$.

(c) There are a non-selfloop arc $a$ and a vertex $w \notin X$ such that $\partial a=v-w$.

(II) For some $v \in Y$ we have one of the following three:

(a) There is a selfloop $a$ such that $\partial a=-2 v$.

(b) There are a non-selfloop arc $a$ and a vertex $w \notin X$ such that $\partial a=-v-w$.

(c) There are a non-selfloop arc $a$ and a vertex $w \notin Y$ such that $\partial a=-v+w$.

Case (I-a) is impossible since $\mathrm{F}(+v) \neq(\emptyset, \emptyset)$ for $v \in X$. In Case (I-b), we have $w \in \mathrm{F}(+v)^{-}$ or $v \in \mathrm{F}(+w)^{-}$. But $w \in \mathrm{F}(+v)^{-}$is impossible from (19) since $w \notin Y$. So, we must have $v \in \mathrm{F}(+w)^{-}$, which implies $w \in \mathrm{F}(+v)^{-} \subseteq Y$ (due to Lemma 4.3 and (19)), a contradiction. Similarly as Case (I-b), Case (I-c) also leads us to a contradiction. Case (II) can be treated similarly as Case (I).

Consequently, $(X, Y)$ must be an ideal of $\mathcal{P}(\mathcal{F})$.

Theorem 4.7 asserts that $\mathcal{P}(\cdot)$ defines an injection from the set of simple signed ring families, on $V$, containing $(\emptyset, \emptyset)$ to the set of signed posets on $V$. We show that the mapping is also onto.

For a signed poset $\mathcal{P}=(V, A ; \partial)$ and a vertex $v \in V$, when $2 v \notin \partial A$, define

$$
\mathrm{I}(+v)=(\{w \mid v-w \in \partial A\} \cup\{v\},\{w \mid v+w \in \partial A\})
$$

and when $-2 v \notin \partial A$, define

$$
\mathrm{I}(-v)=(\{w \mid-v-w \in \partial A\},\{w \mid-v+w \in \partial A\} \cup\{v\}) .
$$

Also, if $2 v \in \partial A$ (or $-2 v \in \partial A$ ), we define $\mathrm{I}(+v)=(\emptyset, \emptyset)$ (or $\mathrm{I}(-v)=(\emptyset, \emptyset)$ ). We can easily see that $\mathrm{I}(+v)$ and $\mathrm{I}(-v)$ are ideals of $\mathcal{P}$ and we call $\mathrm{I}(+v)$ the positive principal ideal at $v$ and $\mathrm{I}(-v)$ the negative principal ideal at $v$ of $\mathcal{P}$. (In fact, for a simple signed ring family $\mathcal{F}$ on $V$ and its corresponding signed poset $\mathcal{P}$ on $V$ we have $\mathrm{I}(+v)=\mathrm{F}(+v)$ and $\mathrm{I}(-v)=\mathrm{F}(-v)$ for $v \in V$. $)$

Lemma 4.8. Let $\mathcal{I}(\mathcal{P})$ be the set of all the ideals of a signed poset $\mathcal{P}$ on $V$. Then $\mathcal{I}(\mathcal{P})$ is a simple signed ring family on $V$ with $(\emptyset, \emptyset) \in \mathcal{I}(\mathcal{P})$. 
(Proof) First, we show that $\mathcal{I}(\mathcal{P})$ is $\sqcup, \sqcap$-closed. Let $\left(X_{i}, Y_{i}\right)(i=1,2)$ be ideals of $\mathcal{P}$. Let us consider their intersection $\left(X_{1}, Y_{1}\right) \sqcap\left(X_{2}, Y_{2}\right)$. For any $a \in A$, if

$$
\left(\partial^{+} a \cap\left(X_{1} \cap X_{2}\right)\right) \cup\left(\partial^{-} a \cap\left(Y_{1} \cap Y_{2}\right)\right) \neq \emptyset,
$$

then we have

$$
\left(\partial^{+} a \cap Y_{1}\right) \cup\left(\partial^{-} a \cap X_{1}\right) \neq \emptyset, \quad\left(\partial^{+} a \cap Y_{2}\right) \cup\left(\partial^{-} a \cap X_{2}\right) \neq \emptyset
$$

since $\left(X_{i}, Y_{i}\right)(i=1,2)$ are ideals. Since we do not have $\partial^{-} a \cap X_{1} \neq \emptyset$ and $\partial^{+} a \cap Y_{2} \neq \emptyset$ (or $\partial^{+} a \cap Y_{1} \neq \emptyset$ and $\partial^{-} a \cap X_{2} \neq \emptyset$ ) due to (23), it follows from (24) that we have $\partial^{+} a \cap\left(Y_{1} \cap Y_{2}\right) \neq \emptyset$ or $\partial^{-} a \cap\left(X_{1} \cap X_{2}\right) \neq \emptyset$, i.e.,

$$
\left(\partial^{+} a \cap\left(Y_{1} \cap Y_{2}\right)\right) \cup\left(\partial^{-} a \cap\left(X_{1} \cap X_{2}\right)\right) \neq \emptyset .
$$

Hence, $\left(X_{1}, Y_{1}\right) \sqcap\left(X_{2}, Y_{2}\right)$ is an ideal of $\mathcal{P}$. Let us now consider the reduced union $\left(X_{1}, Y_{1}\right) \sqcup$ $\left(X_{2}, Y_{2}\right)$. Suppose that for an arc $a \in A$

$$
\left(\partial^{+} a \cap\left(\left(X_{1} \cup X_{2}\right) \backslash\left(Y_{1} \cup Y_{2}\right)\right)\right) \cup\left(\partial^{-} a \cap\left(\left(Y_{1} \cup Y_{2}\right) \backslash\left(X_{1} \cup X_{2}\right)\right) \neq \emptyset .\right.
$$

Then we have $\left(\partial^{+} a \cap Y_{1}\right) \cup\left(\partial^{-} a \cap X_{1}\right) \neq \emptyset$ or $\left(\partial^{+} a \cap Y_{2}\right) \cup\left(\partial^{-} a \cap X_{2}\right) \neq \emptyset$, i.e.,

$$
\left(\partial^{-} a \cap\left(X_{1} \cup X_{2}\right)\right) \cup\left(\partial^{+} a \cap\left(Y_{1} \cup Y_{2}\right)\right) \neq \emptyset,
$$

since $\left(X_{i}, Y_{i}\right)(i=1,2)$ are ideals. If $\partial^{+} a \cap\left(\left(X_{1} \cup X_{2}\right) \cap\left(Y_{1} \cup Y_{2}\right)\right) \neq \emptyset$ (or $\partial^{-} a \cap$ $\left.\left(\left(X_{1} \cup X_{2}\right) \cap\left(Y_{1} \cup Y_{2}\right)\right) \neq \emptyset\right)$, we must have $\partial^{-} a \cap\left(\left(X_{1} \cup X_{2}\right) \backslash\left(Y_{1} \cup Y_{2}\right)\right) \neq \emptyset$ (or $\left.\partial^{+} a \cap\left(\left(Y_{1} \cup Y_{2}\right) \backslash\left(X_{1} \cup X_{2}\right)\right) \neq \emptyset\right)$ due to (26). Hence, from (27) we have

$$
\left(\partial^{-} a \cap\left(\left(X_{1} \cup X_{2}\right) \backslash\left(Y_{1} \cup Y_{2}\right)\right)\right) \cup\left(\partial^{+} a \cap\left(\left(Y_{1} \cup Y_{2}\right) \backslash\left(X_{1} \cup X_{2}\right)\right) \neq \emptyset .\right.
$$

Therefore, $\left(X_{1}, Y_{1}\right) \sqcup\left(X_{2}, Y_{2}\right)$ is an ideal.

Next, we show that $\mathcal{I}(\mathcal{P})$ is spanning. By the definition of the signed poset $\mathcal{P}=(V, A ; \partial)$, for any $v \in V$ we have $2 v \notin \partial A$ or $-2 v \notin \partial A$. Therefore, there exists an ideal $\mathrm{I}(+v) \neq(\emptyset, \emptyset)$ or $\mathrm{I}(-v) \neq(\emptyset, \emptyset)$ for any $v \in V$. Hence from from Lemma 2.1 that every $v \in V$ belongs to the support of $\mathcal{I}(\mathcal{P})$, i.e., $\mathcal{I}(\mathcal{P})$ is spanning.

Finally, we show that $\mathcal{I}(\mathcal{P})$ is simple. For any distinct $v, w \in V$, suppose $2 v \notin \partial A$ without loss of generality. If $w \notin \mathrm{I}(+v)^{+} \cup \mathrm{I}(+v)^{-}$, then we are done and if $w \in \mathrm{I}(+v)^{+} \cup \mathrm{I}(+v)^{-}$, then $\left(\mathrm{I}(+v)^{+} \backslash\{v\}, \mathrm{I}(+v)^{-}\right)$is a desired ideal that separates $v$ and $w$.

We conclude the proof by noting that $(\emptyset, \emptyset)$ is also an ideal of $\mathcal{P}$.

Lemma 4.9. For two signed posets $\mathcal{P}=(V, A ; \partial)$ and $\mathcal{P}^{\prime}=\left(V, A^{\prime} ; \partial^{\prime}\right)$ on $V$, if $\partial A \neq \partial^{\prime} A^{\prime}$, then $\mathcal{I}(\mathcal{P}) \neq \mathcal{I}\left(\mathcal{P}^{\prime}\right)$.

(Proof) If there exists some $v \in V$ such that $2 v \in \partial A \backslash \partial^{\prime} A^{\prime}$ (or $-2 v \in \partial A \backslash \partial^{\prime} A^{\prime}$ ), then the positive (or negative) principal ideal at $v$ of $\mathcal{P}^{\prime}$ is not contained in $\mathcal{I}(\mathcal{P})$. So, suppose that $\mathcal{P}$ and $\mathcal{P}^{\prime}$ have the same set of selfloops. If there exist distinct $v, w \in V$ such that $v-w \in \partial A \backslash \partial^{\prime} A^{\prime}$, then $2 v \notin \partial^{\prime} A^{\prime}$ or $-2 w \notin \partial^{\prime} A^{\prime}$. If $2 v \notin \partial^{\prime} A^{\prime}$ (or $-2 w \notin \partial^{\prime} A^{\prime}$ ), then for the positive (or negative) principal ideal $W$ at $v$ (or $w$ ) of $\mathcal{P}^{\prime}$ we have $w \notin W^{+}$(or $v \notin W^{-}$). Hence, $W$ is not an ideal of $\mathcal{P}$. Other cases are treated similarly. 
From Theorem 4.7, Lemma 4.8, and Lemma 4.9 we have the following theorem. This is a signed-set version of the Birkhoff theorem of Reiner [32], described in terms of signed ring family.

Theorem 4.10 (Reiner). There exists a one-to-one correspondence between the set of all the simple signed ring families $\mathcal{F} \subseteq 3^{V}$ on $V$ with $(\emptyset, \emptyset) \in \mathcal{F}$ and the set of all the signed posets on $V$ such that each such $\mathcal{F}$ is the set of all the ideals of the corresponding signed poset $\mathcal{P}$. In fact, such a one-to-one correspondence is obtained by making each $\mathcal{F}$ correspond to $\mathcal{P}(\mathcal{F})$.

\section{General Signed Ring Families}

In this section we investigate the representation of non-simple and/or non-spanning signed ring families, which is important when we are given such a signed ring family and want to decompose the associated discrete system into subsystems with a signed poset structure on it. The decomposition of signed ring families described in this section is the signed-set counterpart of the Birkhoff-Iri decomposition (Theorem 1.1) of ring families (distributive set lattices).

Consider any signed ring family $\mathcal{F} \subseteq 3^{V}$. Recall that because of Lemma 2.1 we can restrict the underlying set $V$ to its support. Hence we assume that $\mathcal{F}$ is spanning.

Moreover, if a signed ring family on $V$ does not contain $(\emptyset, \emptyset)$, then for the minimum element $\left(X_{0}, Y_{0}\right)$ of $\mathcal{F}$ define

$$
\mathcal{F}^{\prime}=\left\{\left(X \backslash X_{0}, Y \backslash Y_{0}\right) \mid(X, Y) \in \mathcal{F}\right\} .
$$

Then $\mathcal{F}^{\prime}$ is a signed ring family on $V^{\prime}=V \backslash\left(X_{0} \cup Y_{0}\right)$ with $(\emptyset, \emptyset) \in \mathcal{F}^{\prime}$. Hence we can assume that $(\emptyset, \emptyset) \in \mathcal{F}$.

Now, consider any spanning signed ring family $\mathcal{F} \subseteq 3^{V}$ with $(\emptyset, \emptyset) \in \mathcal{F}$. Define an equivalence relation $\sim$ on $V$ as follows. For any $v, w \in V$ we have $v \sim w$ if and only if for each $(X, Y) \in \mathcal{F}$ either $v, w \in X \cup Y$ or $v, w \notin X \cup Y$. The equivalence classes associated with the equivalence relation $\sim$ give a partition $\Pi(\mathcal{F})$ of $V$. By the definition of the equivalence relation we see that each component $K \in \Pi(\mathcal{F})$ is divided into two sets $K_{1}$ and $K_{2}$ (either but not both possibly empty) such that for each $(X, Y) \in \mathcal{F}$ with $K \subseteq X \cup Y$ we have either

(i) $K_{1} \subseteq X$ and $K_{2} \subseteq Y$, or

(ii) $K_{1} \subseteq Y$ and $K_{2} \subseteq X$.

Therefore, we should consider $\Pi(\mathcal{F})$ as a signed partition, where each component $K \in \Pi(\mathcal{F})$ is further partitioned into two sets $K_{1}$ and $K_{2}$ and is identified with a signed set $\left(K_{1}, K_{2}\right) \in$ $3^{V}$. More precisely, we consider as follows.

- For each $K \in \Pi(\mathcal{F})$ the bi-partiton $\left\{K_{1}, K_{2}\right\}$ is unique, and we choose an ordered pair $\left(K_{1}, K_{2}\right)$ and fix it for each $K \in \Pi(\mathcal{F})$ throughout the argument given below. Consider $\Pi(\mathcal{F})$ as a signed partition $\left\{\left(K_{1}, K_{2}\right) \mid K \in \Pi(\mathcal{F})\right\}$ of $V$.

We call $\left(K_{2}, K_{1}\right)$ a reflection of $\left(K_{1}, K_{2}\right)$. We show a way of representing a given spanning signed ring family $\mathcal{F}$ with $(\emptyset, \emptyset) \in \mathcal{F}$ in this section (Theorem 5.1), which is unique up to reflections of components of the signed partition $\left\{\left(K_{1}, K_{2}\right) \mid K \in \Pi(\mathcal{F})\right\}$ of $V$. For each $(X, Y) \in \mathcal{F}$ define a signed set $(\hat{X}, \hat{Y}) \in 3^{\Pi(\mathcal{F})}$ by

$$
(\hat{X}, \hat{Y})=\left(\left\{K \in \Pi(\mathcal{F}) \mid\left(K_{1}, K_{2}\right) \sqsubseteq(X, Y)\right\},\left\{K \in \Pi(\mathcal{F}) \mid\left(K_{2}, K_{1}\right) \sqsubseteq(X, Y)\right\}\right)
$$


and put

$$
\hat{\mathcal{F}}=\{(\hat{X}, \hat{Y}) \mid(X, Y) \in \mathcal{F}\} .
$$

We can easily show that $\hat{\mathcal{F}}$ is a simple signed ring family on the signed partition $\Pi(\mathcal{F})$. We call $\hat{\mathcal{F}}$ a simplification of $\mathcal{F}$. Hence $\hat{\mathcal{F}}$ is the collection of ideals of a unique signed poset $\mathcal{P}(\hat{\mathcal{F}})$, due to Theorem 4.10 .

Conversely, each $(\hat{X}, \hat{Y}) \in \hat{\mathcal{F}}$ is made to correspond to $(X, Y) \in \mathcal{F}$ as follows.

$$
(X, Y)=\sqcup\left(\left\{\left(K_{1}, K_{2}\right) \mid K \in \hat{X}\right\} \cup\left\{\left(K_{2}, K_{1}\right) \mid K \in \hat{Y}\right\}\right) .
$$

We can see that once every signed component $\left(K_{1}, K_{2}\right) \in \Pi(\mathcal{F})$ is fixed, the correspondence between $(X, Y) \in \mathcal{F}$ and $(\hat{X}, \hat{Y}) \in \hat{\mathcal{F}}$ is one-to-one.

We now have our main theorem as follows.

Theorem 5.1 (Reiner-AF). For any spanning family $\mathcal{F}$ of signed sets in $3^{V}$ with $(\emptyset, \emptyset) \in \mathcal{F}$, the family $\mathcal{F}$ is a signed ring family if and only if there exist a signed partition $\Pi(\mathcal{F})$ of $V$ and a signed poset $\mathcal{P}$ on the signed partition $\Pi(\mathcal{F})$ such that for every $(X, Y) \in \mathcal{F}$ there exists an ideal $(\hat{X}, \hat{Y})$ of $\mathcal{P}$ such that (30) and (31) hold.

This is a signed set-theoretical extension of Reiner's theorem [32]. The relation between the present theorem and Reiner's is the signed-set counterpart of the relation between the Birkhoff-Iri theorem and the Birkhoff theorem. Theorem 5.1 furnishes the decomposition of bidirected graphs into strongly connected components with signed poset structures ([4]), which further yields the Edmonds-Gallai decomposition of undirected graphs ([26, 27, 29]).

As is remarked above, for a spanning signed ring family $\mathcal{F} \subseteq 3^{V}$ with $(\emptyset, \emptyset) \in \mathcal{F}$ the corresponding representation by a signed poset on a signed partition $\Pi(\mathcal{F})$ stated in Theorem 5.1 is not unique in general. It is uniquely determined up to the choice of signed components or ordered bi-partitions $\left(K_{1}, K_{2}\right)$ of components $K \in \Pi(\mathcal{F})$, i.e., up to their reflections.

Example 2: See Figure 5 for two signed posets with signed partitions that represent the same signed ring family $\mathcal{F} \subset 3^{\{1,2,3,4,5,6\}}$. Note that the signed component $r=(\{6\},\{5\})$ in Figure 5 (b) is reflected from $r=(\{5\},\{6\})$ in Figure 5 (a) and at the same time the signs of arcs incident to $r$ are reflected. For example, the representation in Figure 5(a) has the set of maximal signed sets (in the simplification of $\mathcal{F}$ ) given by

$$
(\{q, r\},\{p\}), \quad(\{q\},\{p, r\}), \quad(\emptyset,\{p, q, r\}),
$$

while the one in Figure 5(b) has the set of maximal signed sets given by

$$
(\{q\},\{p, r\}), \quad(\{q, r\},\{p\}), \quad(\{r\},\{p, q\}) .
$$

Then, both (33) and (33) give the same set of maximal signed sets in $\mathcal{F}$ as

$$
(\{3,4,5\},\{1,2,6\}), \quad(\{3,4,6\},\{1,2,5\}), \quad(\{3,6\},\{1,2,4,5\}) .
$$




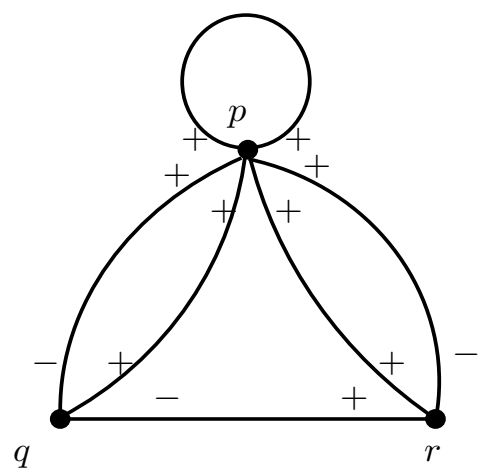

(a)

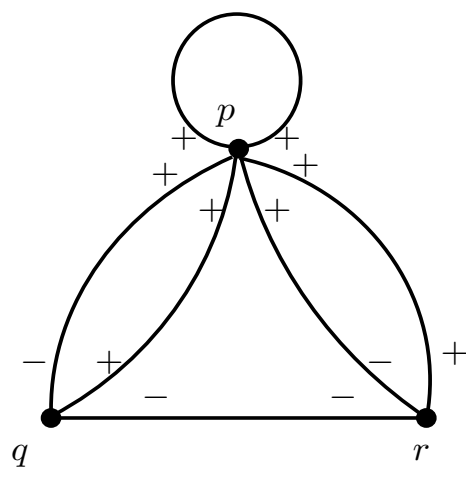

(b)

Figure 5. (a) A signed poset representation and (b) its equivalent representation, where the vertices $p, q, r$ are associated with signed set components: in Figure 3(a): $p=(\{1,2\},\{3\}), q=(\{4\}, \emptyset)$, and $r=(\{5\},\{6\})$, while in Figure 3(b): $p=(\{1,2\},\{3\}), q=(\{4\}, \emptyset)$, and $r=(\{6\},\{5\})$.

\section{Concluding Remarks}

Although the original version [1] of this paper has been left unpublished, the signed-set decomposition of signed ring families shown in Sections 4 and 5 has played crucial roles for minimizing bisubmodular functions in (strongly) polynomial time in $[12,17,30]$, decomposition of skew-symmetric matrices in [26, 27], and the principal partition of bisubmodular systems in [16]. Since the bisubmodularity and its extensions have recently drawn much attention of researchers (see, e.g., $[12,15,18,19])$, the detailed discussions and proofs given in the present paper will be useful for further investigation on the related research subjects.

\section{Acknowledgments}

K. Ando's work was partly supported by JSPS KAKENHI Grant Number 18 K11180 and S. Fujishige's by JSPS KAKENHI Grant Number 19K11839. The authors' original work [1] was made while the second author was visiting Research Institute for Discrete Mathematics, University of Bonn, as an Alexander-von-Humboldt fellow hosted by Professor Bernhard Korte, which they gratefully acknowledge.

\section{References}

[1] K. Ando and S. Fujishige: $\sqcup, \sqcap$-closed families and signed posets. Report No. 93813, Research Institute for Discrete Mathematics, University of Bonn, January 1994.

[2] K. Ando and S. Fujishige: On structures of bisubmodular polyhedra. Mathematical Programming 74 (1996) 293-318.

[3] K. Ando, S. Fujishige, and T. Naitoh: Balanced bisubmodular systems and bidirected flows. Journal of the Operations Research Society of Japan 40 (1997) 437-447.

[4] K. Ando, S. Fujishige, and T. Nemoto: Decomposition of a signed graph into strongly connected components and its signed poset structure. Discrete Applied Mathematics 68 (1996) 237-248.

[5] J. M. Bilbao, J. R. Fernández, N. Jiménez, and J. J. López: Biprobabilistic values for bicooperative games. Discrete Applied Mathematics 156 (2008) 2698-2711.

[6] G. Birkhoff: Rings of sets. Duke Mathematical Journal 3 (1937) 443-454. 
[7] G. Birkhoff: Lattice Theory (American Mathematical Colloquium Publications 25 (3rd ed.), Providence, R. I., 1967).

[8] A. Bouchet and W. H. Cunningham: Delta-matroids, jump systems and bisubmodular polyhedra. SIAM Journal on Discrete Mathematics 8 (1995) 17-32.

[9] R. Chandrasekaran and S. N. Kabadi: Pseudomatroids. Discrete Mathematics 71 (1988), 205-217.

[10] F. D. J. Dunstan and D. J. A. Welsh: A greedy algorithm for solving a certain class of linear programmes. Mathematical Programming 62 (1973) 338-353.

[11] K. Fujimoto, T. Murofushi, and M. Sugeno: $k$-additivity and $\mathcal{C}$-decomposability of bi-capacities and its integral. Fuzzy Sets and Systems 158 (2007) 1698-1712.

[12] S. Fujishige: A min-max theorem for bisubmodular polyhedra. SIAM Journal on Discrete Mathematics 10 (1997) 294-308.

[13] S. Fujishige: Submodular Functions and Optimization (North-Holland, Amsterdam, 1991), Second Edition (Elsevier, 2005)

[14] S. Fujishige: Theory of principal partitions revisited. Research Trends in Combinatorial Optimization (W. Cook, L. Lovász, and J. Vygen, eds., Springer, Berlin, 2009), pp. 127-162.

[15] S. Fujishige: Bisubmodular polyhedra, simplicial divisions, and discrete convexity. Discrete Optimization 12 (2014) 115-120.

[16] S. Fujishige: Parametric bisubmodular function minimization and its associated signed ring family. Discrete Applied Mathematics 227 (2017) 142-148.

[17] S. Fujishige and S. Iwata: Bisubmodular function minimization. SIAM Journal on Discrete Mathematics 19 (2006) 1065-1073.

[18] S. Fujishige and S. Tanigawa: Polynomial combinatorial algorithms for skew-bisubmodular function minimization. Mathematical Programming, Ser. A, 171 (2018) 87-114

[19] S. Fujishige, S. Tanigawa, and Y. Yoshida: Generalized skew bisubmodularity: A characterization and a min-max theorem. Discrete Optimization 12 (2014) 1-9.

[20] M. Grabisch and C. Labreuche: Bi-capacities. I. Definition, Möbius transform and interaction. Fuzzy Sets and Systems 151 (2005) 211-236.

[21] M. Grabisch and C. Labreuche: Bi-capacities. II. The Choquet integral. Fuzzy Sets and Systems 151 (2005) 237-259.

[22] M. Iri: The maximum-rank minimum-term-rank theorem for the pivotal transforms of a matrix. Linear Algebra and Its Applications 2 (1969) 427-446.

[23] M. Iri: A review of recent work in Japan on principal partitions of matroids and their applications. Annals of the New York Academy of Sciences 319 (1979) 306-319.

[24] M. Iri: Applications of matroid theory. Mathematical Programming-The State of the Art (A. Bachem, M. Grötschel and B. Korte, eds., Springer, Berlin, 1983), pp. 158-201.

[25] M. Iri: Structural theory for the combinatorial systems characterized by submodular functions. In: Progress in Combinatorial Optimization (W. R. Pulleyblank, ed., Academic Press, Toronto, 1984), pp. 197-219.

[26] S. Iwata: Dulmage-Mendelsohn type decomposition for general graphs. RIMS Preprint, RIMS1017, Kyoto University, May 1995.

[27] S. Iwata: Block triangularization of skew-symmetric matrices. Linear Algebra and Its Applications 273 (1998) 215-226.

[28] G. Kishi and Y. Kajitani: Maximally distant trees in a linear graphs (in Japanese). Transactions of the Institute of Electronics and Communication Engineers of Japan 51A (1968) 196-203; also see: On maximally distinct trees. Proceedings of the Fifth Annual Allerton Conference on Circuit and System Theory (October 4-6, 1967), pp. 635-643.

[29] L. Lovász and M. D. Plummer: Matching Theory (AMS Chelsea Publishing, 1986).

[30] S. T. McCormick and S. Fujishige: Strongly polynomial and fully combinatorial algorithms for bisubmodular function minimization. Mathematical Programming, Ser. A 122 (2010) 87-120.

[31] L. Qi: Directed submodularity, ditroids and directed submodular flows. Mathematical Programming 42 (1988) 579-599.

[32] V. Reiner: Signed posets. Journal of Combinatorial Theory, Series A 62 (1993), 324-360. 